\title{
Genetic analysis of common variants in the HDAC2 gene with schizophrenia susceptibility in Han Chinese
}

\author{
Gang Chen ${ }^{1,2,5}$, Fanglin Guan ${ }^{1,2,3,5}$, Huali $\mathrm{Lin}^{4}, \mathrm{Lu} \mathrm{Li}^{1,2}$ and Dongke $\mathrm{Fu}^{2}$
}

Schizophrenia (SCZ) is a complex psychiatric disorder that is strongly influenced by a genetic component. Recent studies suggested that histone deacetylases (HDACs) might increase the expression of several key genes in the brain and may also be associated with susceptibility to SCZ. Among human HDACs, HDAC2 is a critical modulator of gene regulation. Here, we designed a two-stage case-control study to thoroughly examine the association between the HDAC2 gene and SCZ. A total of 19 common single-nucleotide polymorphisms (SNPs) in the region of the HDAC2 gene were analyzed in the test group of 1430 patients and $\mathbf{2 8 6 2}$ matched healthy controls. A comparison of the genotype and allele frequencies of the SNPs between cases and controls revealed that three SNPs, rs13212283, rs6568819 and rs9488289, were nominally associated with SCZ. However, we failed to observe any association between these SNPs and SCZ in the validation group consisting of 896 cases and 1815 matched healthy controls. Furthermore, haplotypic analysis also confirmed the negative results. Our results provide preliminary evidence that HDAC2 may not confer susceptibility to SCZ in Han Chinese. Additional genetic studies from a large population are required to obtain more conclusive results.

Journal of Human Genetics (2015) 60, 479-484; doi:10.1038/jhg.2015.66; published online 11 June 2015

\section{INTRODUCTION}

Schizophrenia (SCZ) is a complex mental disorder and is one of the most debilitating psychiatric disorders, affecting $\sim 1.1 \%$ of the general population over the age of 18 worldwide. ${ }^{1}$ Although there is increasing evidence to show that both environmental and genetic factors have important roles in the occurrence and development of SCZ, ${ }^{2-4}$ the etiology and genetic mechanisms of SCZ remain unknown. ${ }^{5}$ Early genetic epidemiological studies indicated that heritability in the development of SCZ was estimated to reach $80 \%{ }^{6}$ Recent genetic studies of association mapping demonstrated substantial polygenic components contributing to SCZ susceptibility, ${ }^{7-10}$ and these studies have been accelerated by the widely used application of genome-wide association studies on SCZ. ${ }^{11}$ However, genome-wide association studies provide a promising approach for studying the genetics of SCZ, but as a result of the genetic heterogeneity of SCZ in different populations, the identified genetic susceptibility loci for the disease has often failed to be replicated across studies. ${ }^{12}$

Epigenetic modification is a mechanism underlying the relationship between environmental exposure and the individual genetic background, and emerging evidence from several studies has shown that epigenetic phenomena might be an explanation of the etiology of SCZ. ${ }^{13}$ DNA methylation and covalent modifications of histone tails are hypothesized to be two important regulatory mechanisms that cause heritable changes in gene expression without DNA sequence variation. The expression of many functional genes in the human nervous system can be precisely regulated by DNA methylation. ${ }^{14}$ Previous studies reported that abnormal gene methylation has a key role in the development of SCZ. ${ }^{15,16}$ For the covalent modification of histones, histone acetylation/deacetylation is the primary epigenetic mechanism regulating gene expression, which is catalyzed by two key enzymes, histone acetyltransferase and histone deacetylase (HDAC). ${ }^{17}$ HDACs may increase the expression of several key genes in the brain and may also be associated with susceptibility to SCZ. ${ }^{18,19}$ Among human HDACs, HDAC2 is a critical modulator of the regulation of cell proliferation, and also has an important role in gene regulation because it catalyzes a series of key reactions in physiological processes. ${ }^{20}$ Therefore, the HDAC2 gene is a promising candidate gene for SCZ. Although there has been a previous study of the association between the HDAC2 gene and SCZ in the Han Chinese, only four single-nucleotide polymorphisms (SNPs) in a small family sample were analyzed. ${ }^{21}$ Because of the additive effect of many minor genetic contributions to disease occurrence and because of the unknown biological mechanisms of HDAC2 involved in the pathophysiology of SCZ, it remains necessary to systematically explore the potential association between HDAC2 polymorphisms and SCZ in a larger random case-control sample.

In our study, to further investigate whether common variants in the HDAC2 gene confer some risk of susceptibility to SCZ, we conducted

${ }^{1}$ Department of Forensic Psychiatry, School of Medicine \& Forensics, Xi'an Jiaotong University, Xi'an, China; ${ }^{2}$ Key Laboratory of National Ministry of Health for Forensic Sciences, School of Medicine \& Forensics, Xi'an Jiaotong University, Xi'an, China; ${ }^{3}$ Key Laboratory of Environment and Genes Related to Diseases, Ministry of Education, Xi'an, China and ${ }^{4}$ Department of Psychiatry, Xi'an Mental Health Center, Xi'an, Shaanxi, China

5 These authors contributed equally to this work.

Correspondence: Dr F Guan, Department of Forensic Psychiatry, School of Medicine \& Forensics, Xi'an Jiaotong University, 76 West Yanta Road, Xi'an 710061, China. E-mail: fanglingguan@163.com

Received 15 March 2015; revised 1 May 2015; accepted 3 May 2015; published online 11 June 2015 
a large-sample two-stage association analysis in case-control subjects from the Han Chinese population.

\section{MATERIALS AND METHODS}

\section{Subjects}

Two separate data sets were included in this study, and a two-stage approach was utilized for the discovery and for single marker analyses. The subjects were 1430 SCZ cases (764 males, mean age $=34.4 \pm 7.07 ; 666$ females, mean age $=35.8 \pm 6.81)$ and 2862 healthy controls (1520 males, mean age $=$ $36.2 \pm 6.91 ; 1342$ females, mean age $=38.3 \pm 6.46$ ), which were the testing set; $896 \mathrm{SCZ}$ cases ( 474 males, mean age $=33.8 \pm 7.43 ; 422$ females, mean age $=$ $34.6 \pm 7.92)$ and 1815 healthy controls (942 males, mean age $=35.7 \pm 7.74$; 873 females, mean age $=36.3 \pm 7.46)$ were categorized as the validation set. All patients were recruited from the inpatient and outpatient clinical services of a psychiatric unit at Xi'an Mental Health Center, and all unrelated healthy controls were local volunteers. In patient samples, all diagnoses were assigned by a standard procedure and confirmed by at least two experienced psychiatrists using the Structured Clinical Interview for Diagnostic and Statistical Manual of Mental Disorders, 4th Revision Axis I disorder and the Diagnostic and Statistical Manual of Mental Disorders, 4th Revision. Patients with substanceinduced psychotic disorders, learning disabilities, head injuries and other symptomatic psychoses were excluded from the present study. Moreover, the patients reported not taking any antischizophrenic drug for at least 1 week before they entered into this study. Healthy subjects were enrolled from the city of Xi'an in Shaanxi Province. All subjects are of Han descent, and based on self-report regarding their own and paternal grandparents' place of birth, we excluded anyone not born locally or whose families were not born locally for three generations. All participants provided written informed consent. The Ethics Committee of Xi'an Jiaotong University approved this study.

\section{SNP selection and genotyping}

As an initial screen of common SNPs in the Han Chinese population, we searched for all SNPs with minor allele frequencies $\geqslant 0.01$ between $15 \mathrm{~kb}$ upstream and $15 \mathrm{~kb}$ downstream (30-kb window) of the HDAC2 gene in the HapMap HCB data set (HapMap Data Version 3 and Release R2), and 19 SNPs were identified, rs2169506, rs2810164, rs352066, rs 13212283, rs538681, rs3757016, rs352068, rs11391, rs9481408, rs13204445, rs12208304, rs6568819, rs10499080, rs9488289, rs13209064, rs2243356, rs2499618, rs2025191 and rs9400686. Therefore, these 19 SNPs, which completely covered the region of the HDAC2 gene, were included in the analyses.

Peripheral venous blood samples were collected in plain tubes and were stored at $-20^{\circ} \mathrm{C}$. Genomic DNA was isolated from peripheral blood leukocytes

Table 1 Single SNP association analysis in the testing data set

\begin{tabular}{|c|c|c|c|c|c|c|c|c|c|}
\hline \multirow{2}{*}{$\begin{array}{l}\text { SNP Markers } \\
\text { rs13212283 }\end{array}$} & \multicolumn{2}{|c|}{ Allele Freq. (\%) } & \multirow[t]{2}{*}{ P-value } & \multicolumn{3}{|c|}{ Genotype Freq. (\%) } & \multirow[t]{2}{*}{ P-value } & \multirow[t]{2}{*}{$H W E$} & \multirow[t]{2}{*}{$O R^{b} 95 \% \mathrm{Cl}$} \\
\hline & G & $A$ & & $\mathrm{GG}$ & $\mathrm{GA}$ & $\mathrm{AA}$ & & & \\
\hline SCZ & $1861(65.07)$ & 999 (34.93) & 0.039440 & 616 (43.08) & 629 (43.99) & 185 (12.94) & 0.043454 & 0.221 & 1.11 \\
\hline CTR & 3852 (67.3) & $1872(32.7)$ & $\underline{0.749} \underline{360}$ & $1288(45)$ & $1276(44.58)$ & $298(10.41)$ & $\underline{0.825} \underline{626}$ & 0.491 & $1.01-1.21$ \\
\hline \multicolumn{10}{|l|}{ Female } \\
\hline SCZ & $870(65.32)$ & 462 (34.68) & 0.103132 & $288(43.24)$ & $294(44.14)$ & 84 (12.61) & 0.213866 & 0.507 & 1.12 \\
\hline CTR & $1822(67.88)$ & $862(32.12)$ & & $617(45.98)$ & $588(43.82)$ & $137(10.21)$ & & 0.859 & $0.98-1.29$ \\
\hline \multicolumn{10}{|l|}{ Male } \\
\hline SCZ & $991(64.86)$ & $537(35.14)$ & 0.195751 & $328(42.93)$ & $335(43.85)$ & $101(13.22)$ & 0.177485 & 0.292 & 1.09 \\
\hline CTR & $2030(66.78)$ & $1010(33.22)$ & & $671(44.14)$ & $688(45.26)$ & $161(10.59)$ & & 0.433 & $0.96-1.24$ \\
\hline rs6568819 & C & $\mathrm{T}$ & & $\mathrm{CC}$ & CT & TT & & & \\
\hline SCZ & 1942 (67.9) & 918 (32.1) & 0.034818 & $648(45.31)$ & $646(45.17)$ & $136(9.51)$ & 0.061140 & 0.169 & 1.11 \\
\hline CTR & $3756(65.62)$ & 1968 (34.38) & $\underline{0.661} \underline{542}$ & $1231(43.01)$ & $1294(45.21)$ & 337 (11.77) & & 0.913 & $1.01-1.22$ \\
\hline \multicolumn{10}{|l|}{ Female } \\
\hline $\mathrm{SCZ}$ & 916 (68.77) & $416(31.23)$ & 0.094431 & $310(46.55)$ & $296(44.44)$ & $60(9.01)$ & 0.211339 & 0.371 & 1.13 \\
\hline CTR & $1775(66.13)$ & 909 (33.87) & & $584(43.52)$ & $607(45.23)$ & $151(11.25)$ & & 0.721 & $0.98-1.30$ \\
\hline \multicolumn{10}{|l|}{ Male } \\
\hline SCZ & $1026(67.15)$ & 502 (32.85) & 0.182699 & $338(44.24)$ & $350(45.81)$ & 76 (9.95) & 0.259882 & 0.289 & 1.09 \\
\hline CTR & $1981(65.16)$ & 1059 (34.84) & & $647(42.57)$ & $687(45.2)$ & $186(12.24)$ & & 0.861 & $0.96-1.25$ \\
\hline rs9488289 & C & G & & $\mathrm{CC}$ & CG & GG & & & \\
\hline SCZ & 1754 (61.33) & 1106 (38.67) & 0.040879 & $551(38.53)$ & $652(45.59)$ & 227 (15.87) & 0.055836 & 0.143 & 1.10 \\
\hline CTR & 3640 (63.59) & $2084(36.41)$ & $\underline{0.776} \underline{701}$ & 1156 (40.39) & $1328(46.4)$ & $378(13.21)$ & & 0.912 & $1.00-1.21$ \\
\hline \multicolumn{10}{|l|}{ Female } \\
\hline SCZ & 822 (61.71) & $510(38.29)$ & 0.142125 & $258(38.74)$ & 306 (45.95) & $102(15.32)$ & 0.272607 & 0.474 & 1.11 \\
\hline CTR & $1720(64.08)$ & 964 (35.92) & & $550(40.98)$ & $620(46.2)$ & $172(12.82)$ & & 0.895 & $0.97-1.27$ \\
\hline \multicolumn{10}{|l|}{ Male } \\
\hline SCZ & 932 (60.99) & $596(39.01)$ & 0.154413 & 293 (38.35) & 346 (45.29) & $125(16.36)$ & 0.197143 & 0.183 & 1.10 \\
\hline CTR & $1920(63.16)$ & $1120(36.84)$ & & 606 (39.87) & $708(46.58)$ & $206(13.55)$ & & 0.972 & $0.97-1.24$ \\
\hline
\end{tabular}

Abbreviations: Cl: confidence interval; CTR: control; HWE, Hardy-Weinberg equilibrium; OR: odds ratio; SCZ: schizophrenia; SNP, single-nucleotide polymorphism.

asignificant $P$-values are in italic bold and corrected $P$-values are underlined after Bonferroni correction.

bOR refers to risk allele odds ratio in cases and controls. 
Table 2 Single SNP association analysis in the validation data set

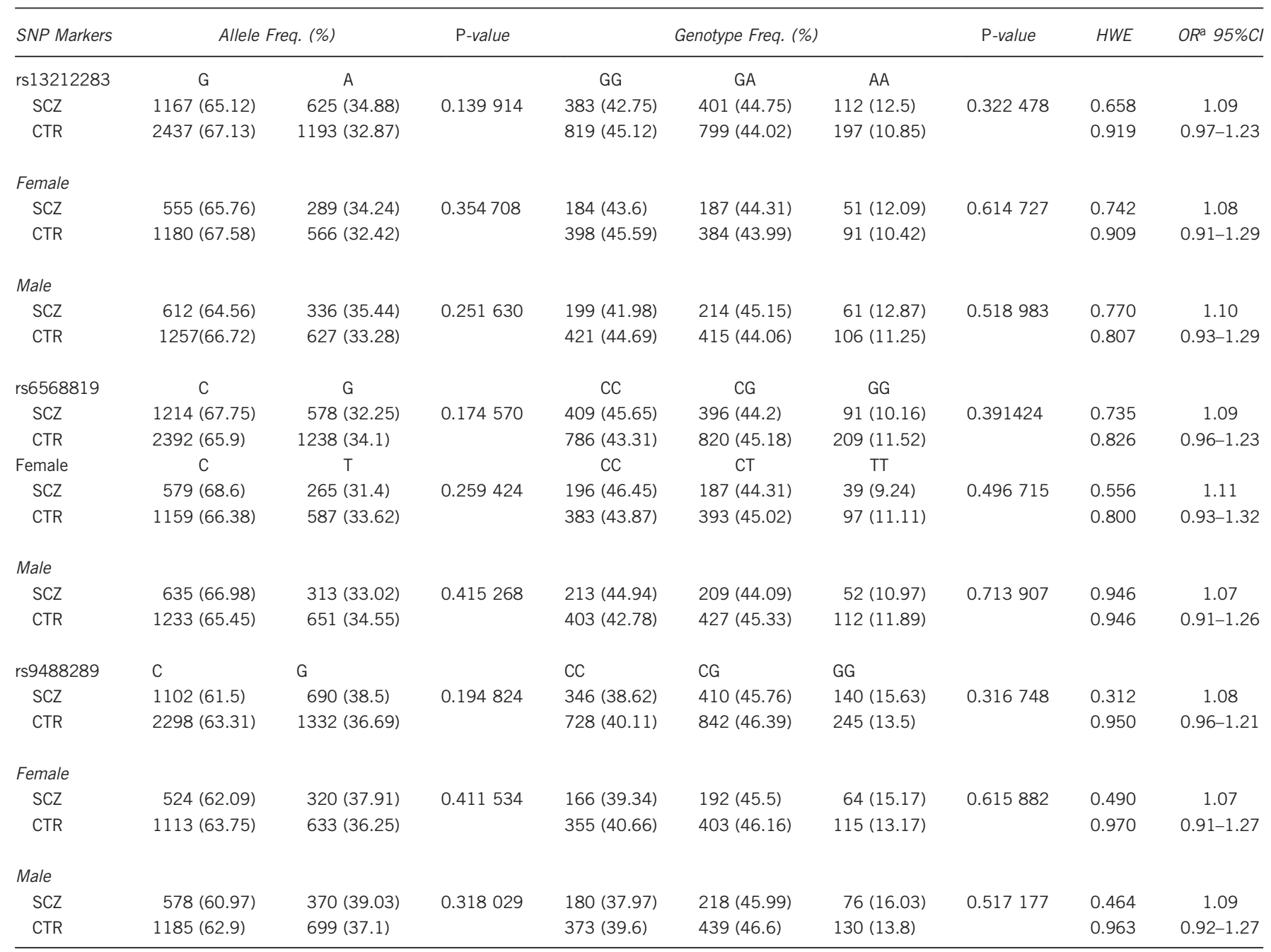

Abbreviations: Cl: confidence interval; CTR: control; HWE, Hardy-Weinberg equilibrium; OR: odds ratio; SCZ: schizophrenia; SNP, single-nucleotide polymorphism.

${ }^{\mathrm{a} O R}$ refers to risk allele odds ratio in cases and controls.

according to the manufacturer's protocol (Genomic DNA kit, Axygen Scientific, CA, USA), and DNA samples were stored at $-20{ }^{\circ} \mathrm{C}$ for SNP analysis. All of the SNPs were genotyped using high-throughput, matrix-assisted laser desorption ionization-time-of-flight mass spectrometry. Briefly, SNPs were genotyped using high-throughput, matrix-assisted laser desorption ionization-time-offlight mass spectrometry. Next, the resulting spectra were processed using Typer Analyzer software (Sequenom, San Diego, CA, USA), and genotype data were generated from the samples. Because the final genotype call rate of each SNP was greater than $99.2 \%$ and the overall genotyping call rate was $99.8 \%$, the reliability of further statistical analysis was ensured.

\section{Statistical analysis}

Haploview v4.2 (Broad Institute of MIT and Harvard, USA. http://www. broadinstitute.org/scientific-community/science/programs/medical-andpopulation-genetics/haploview/haploview) was used to conduct the HardyWeinberg equilibrium test for each SNP in both the case and control groups. Allelic and genotypic association tests were conducted using the genetic analysis software CLUMP v2.4. Haploview v4.2 software program was used to investigate the linkage disequilibrium structure of the candidate markers. To avoid a potential bias caused by minor differences from the haplotype frequency estimates in the heterogeneity test statistics, we implemented score tests that account for the linkage phase ambiguity of the haplotype association. ${ }^{22}$ Permutation was implemented to obtain the maximum haplotype-specific score statistics and their $P$-values. The global $P$-value of the haplotype analysis was calculated based on the differences of the haplotypic frequency distribution among patients and controls. Haplo Stats package v1.6.11 was utilized for these analyses. In addition, we also performed stratification analysis to investigate the potential effects of gender on the single SNP and haplotype association analyses. A $P$-value of 0.05 was selected as the threshold for significance in all analyses. In the study design stage, we calculated the statistical power using PGA v2.0 (National Cancer Institute, USA. http://dceg.cancer.gov/tools/analysis/pga). Our sample size can detect SNP and haplotype associations with 95 and $88 \%$ power, respectively, at a false positive rate of $5 \%$.

\section{RESULTS}

A total of 19 SNPs in the HDAC2 gene were genotyped in the testing data set (1430 SCZ cases and 2862 controls). The allele and genotype distributions of all of the SNPs in both the cases and controls, including the results of the Hardy-Weinberg equilibrium test, are shown in Table 1 and Supplementary Table S1. The distributions were highly polymorphic in both samples, and the allelic and the genotype distributions were all in Hardy-Weinberg equilibrium $(P>0.05)$. As shown in Table 1, when all of the samples were considered in the testing data set, we observed associations for three SNPs (rs13212283, rs6568819 and rs9488289; $P=0.039440,0.034818$ and 0.040879, 


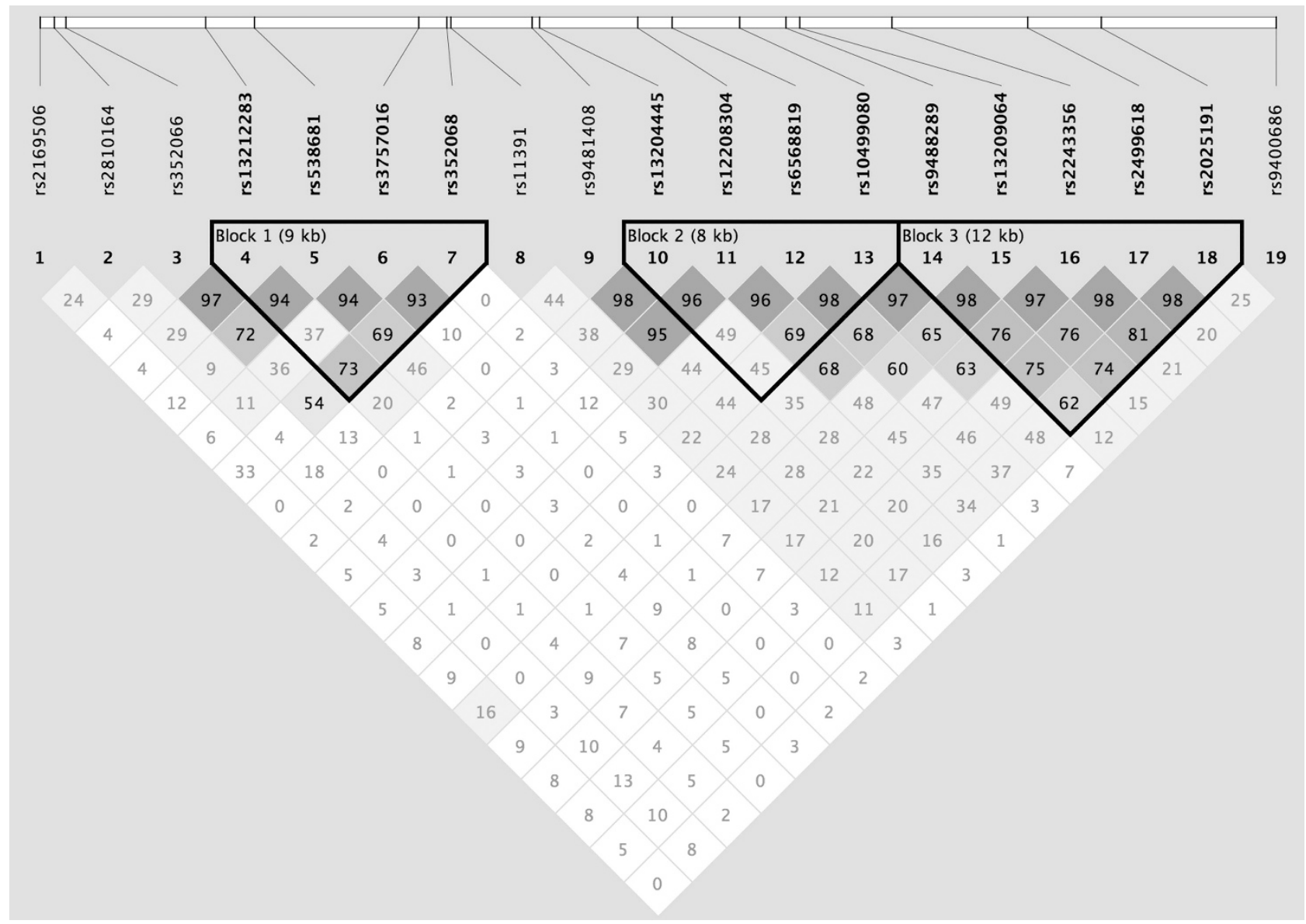

Figure 1 Estimation of linkage disequilibrium between each pair of 19 single-nucleotide polymorphisms genotyped in HDAC2 gene in Han Chinese individuals. Linkage disequilibrium structure (D) between marker pairs was indicated by the shaded matrices. A full color version of this figure is available at the Journal of Human Genetics journal online.

respectively) (Table 1). However, they did not show a significant association with SCZ after the Bonferroni correction (corrected $P=0.749360,0.661542$ and 0.776701 , respectively). Genotypic association analyses confirmed the results. There were no significant differences for the remaining 16 SNPs for both the allelic and genotypic analyses (Supplementary Table S1).

According to the small effect sizes conferred by common alleles requiring the use of large samples, the overall state of a given SNP is best summarized by an association analysis of different populations. Therefore, we performed a single SNP association analysis for the above three SNPs in the validation data set (896 SCZ cases and 1815 controls). However, no significant associations with SCZ were observed (Table 2). To determine whether gender plays a role in the nominal associations, as suggested in the testing data set, we analyzed our data by separating the males and females in the testing and validation data sets. We found no significant association with SCZ in females or males (Tables 1 and 2).

To perform haplotype association analyses, we examined the linkage disequilibrium structure within the genotype data of 19 SNPs and identified three haplotype blocks (Figure 1), which were defined using Haploview's 'confidence intervals' method. ${ }^{23,24}$ Haplotypic association analyses were performed to test possible associations in these linkage disequilibrium blocks. We found that there were no significant association signals (all global $P$-value $>0.05$ ) in these three haplotype blocks (Table 3), and an association was not observed in males or females (all global $P$-value $>0.05$ ) (Table 4). Together with the results of the single SNP association analyses, no evidence of an association with SCZ was observed.

\section{DISCUSSION}

Recently, accumulating evidence has indicated that HDAC inhibitors improve learning and cognitive deficits in animal models, ${ }^{25-27}$ and the roles of HDACs in brain function have been elucidated. ${ }^{28,29}$ Chen et al. found that the expression of the reelin protein was reduced by $50 \%$ in cortical structures of postmortem brains from SCZ patients. Furthermore, reelin protein levels are increased with an HDAC2 inhibitor to inhibit the methylation of the reelin gene promoter in a mouse model of SCZ. ${ }^{30}$ Moreover, a recent report demonstrated that inhibiting HDAC2 might be therapeutic in SCZ. ${ }^{31}$ Therefore, the results from these studies suggest that HDAC2 may be a risk factor for SCZ susceptibility. However, there is not sufficient available genetic data to determine whether HDAC2 is an etiological factor for the development of SCZ.

In our study, our results did not provide evidence for the involvement of HDAC2 in the etiology of SCZ, at least in Han Chinese individuals. Here, we examined 19 SNPs across the genomic region of $50 \mathrm{~kb}$ containing the HDAC2 gene in $1430 \mathrm{SCZ}$ patients and 2826 matched healthy controls, of which three SNPs underwent further validation analyses in an additional independent cohort of 896 cases and 1815 matched controls. However, following the analyses of 
these SNPs, we did not observe any association signals between these SNPs in HDAC2 and SCZ. Indeed, it is not possible to draw a conclusion based only on the analysis of several SNPs. Therefore, we performed a haplotype analysis that supported our negative results. Our findings were consistent with a previous family study in Chinese Han, which reported that there was no observable association between HDAC2 and SCZ. ${ }^{21}$ The molecular mechanism of acetylation regulation is complex, and some genes, pathways and signaling molecules are confirmed to be involved in the process. Because of the complex network of HDAC signaling in the brain and the interactions with

Table 3 Common haplotype frequency and association analysis

\begin{tabular}{lcccccc}
\hline & & & \multicolumn{5}{c}{ Frequency (\%) } \\
\cline { 4 - 7 } ID & LD block & Haplotype & SCZ & CTR & P-value & Global P-value \\
\hline HAP1 & Block 1 & GTCT & 50.1 & 52.0 & 0.085 & \multirow{2}{*}{0.058} \\
HAP2 & & ATCT & 15.3 & 14.7 & 0.459 & \\
HAP3 & & GTTT & 12.9 & 13.7 & 0.335 & \\
HAP4 & & ATTT & 9.68 & 7.88 & 0.048 & \\
HAP5 & & ACTC & 5.02 & 5.18 & 0.488 & \multirow{2}{*}{0.061} \\
HAP6 & Block 2 & TCCC & 55.1 & 55.3 & 0.857 & \\
HAP7 & & CGTT & 13.5 & 13.2 & 0.389 & \\
HAP8 & & CCCC & 11.44 & 9.80 & 0.025 & \\
HAP9 & & TCTT & 8.99 & 10.15 & 0.104 & \\
HAP10 & & CGTC & 3.84 & 4.09 & 0.6 & \\
HAP11 & Block 3 & CATCA & 53.3 & 55.4 & 0.074 & \\
HAP12 & & GCATG & 22.2 & 22.3 & 0.9102 & \\
HAP13 & & GATCA & 9.47 & 7.93 & 0.029 & \\
HAP14 & & GCACA & 4.19 & 4.03 & 0.695 & \\
HAP15 & & CAATG & 3.87 & 3.73 & 0.751 & \\
\hline
\end{tabular}

Abbreviations: CTR, control; LD, linkage disequilibrium; SCZ, schizophrenia.

Block 1: rs13212283-rs538681-rs3757016-rs352068; and Block 2: rs13204445-

rs12208304-rs6568819-rs10499080. Block 3: rs9488289-rs13209064-rs2243356-

rs2499618-rs2025191. Significant $P$-values are in italic bold. Rare haplotypes are not shown,

if frequency $<3 \%$.

a Based on 10000 permutations.

based on comparison of frequency distribution of all haplotypes for the combination of SNPS. neurotransmitter systems, the identification of potential epistatic interactions with HDAC family genes and other genes would further elucidate the roles of HDAC2 in the pathology of SCZ and would also be a more sensitive method to characterize the effects of candidate genes on SCZ.

When interpreting our results, several potential limitations of our study should be noted. First, we could not conclusively rule out other potential confounders, such as clinical symptoms (positive or negative), education duration, medical history and drugs in treatment, which may influence the stability and accuracy of our results. Second, we designed the study based on the 'Common Disease-Common Variant' hypothesis, and we have not yet sequenced the HDAC2 gene to completely evaluate the effect of rare variants on SCZ susceptibility. It is possible that rare variants may contribute to the risk of SCZ in an unpredictably manner or in linkage disequilibrium with other undiscovered markers involved in acetylation regulation that confer a risk for SCZ. Therefore, because of the multiple variants with small effects and the molecular basis of the associations on the complex network underlying the etiology and pathophysiology of SCZ, our study should be considered preliminary and additional follow-up studies are required, including high-density mapping and targeted deep sequencing to undercover the fundamental characteristics of pathogenic HDAC2 mutations and any potential association with SCZ. Finally, although the matching of the population geographics and genetic backgrounds help avoid potential population stratification, we cannot exclude the possibility of a population structure effect in our subjects. Our results must be replicated and validated in other ethnic groups, which would clarify the relationship between HDAC2 and SCZ.

In summary, our results provide preliminary evidence that the HDAC2 gene may not be involved in SCZ susceptibility in the Han Chinese population. Further studies are required to investigate the roles of HDAC2 and other genes from the HDAC family and their interactions to obtain more conclusive results.

Table 4 Gender-specific haplotypes frequency and association analysis

\begin{tabular}{|c|c|c|c|c|c|c|c|c|c|}
\hline \multirow{2}{*}{$\begin{array}{l}L D \\
\text { Block }\end{array}$} & \multirow[b]{2}{*}{$I D$} & \multirow[b]{2}{*}{ Haplotype } & \multicolumn{2}{|c|}{ Female (\%) } & \multicolumn{2}{|c|}{ Male (\%) } & \multicolumn{2}{|c|}{ P-value } & \multirow[b]{2}{*}{ Globato P-value } \\
\hline & & & $S C Z$ & CTR & $S C Z$ & CTR & Female & Male & \\
\hline \multirow[t]{5}{*}{ Block 1} & HAP1 & GTCT & 49.9 & 52.6 & 50.2 & 51.6 & 0.131 & 0.363 & Female \\
\hline & HAP2 & АТCT & 16.4 & 14.8 & 14.4 & 14.5 & 0.219 & 0.906 & 0.259 \\
\hline & HAP3 & GTTT & 13.1 & 14.0 & 12.7 & 13.4 & 0.475 & 0.545 & Male \\
\hline & HAP4 & ATTT & 7.92 & 6.59 & 11.2 & 9.02 & 0.270 & 0.105 & 0.110 \\
\hline & HAP5 & ACTC & 4.45 & 4.98 & 5.45 & 5.36 & 0.415 & 0.442 & \\
\hline \multirow[t]{5}{*}{ Block 2} & HAP6 & TCCC & 55.2 & 55.8 & 55.1 & 54.8 & 0.714 & 0.889 & Female \\
\hline & HAP7 & CGTT & 13.4 & 13.2 & 13.6 & 13.3 & 0.172 & 0.214 & 0.140 \\
\hline & HAP8 & $\mathrm{CCCC}$ & 11.9 & 9.88 & 11.0 & 9.76 & 0.077 & 0.203 & Male \\
\hline & HAP9 & TCTT & 9.45 & 10.9 & 8.57 & 9.50 & 0.201 & 0.347 & 0.164 \\
\hline & HAP10 & CGTC & 3.11 & 3.65 & 4.47 & 4.45 & 0.262 & 0.714 & \\
\hline \multirow[t]{5}{*}{ Block 3} & HAP11 & CATCA & 54.1 & 57.4 & 52.7 & 53.7 & 0.054 & 0.488 & Female \\
\hline & HAP12 & GCATG & 22.4 & 23.2 & 22.0 & 21.5 & 0.163 & 0.172 & 0.226 \\
\hline & HAP13 & GATCA & 9.44 & 7.05 & 9.53 & 8.70 & 0.015 & 0.427 & Male \\
\hline & HAP14 & GCACA & 4.47 & 4.14 & 3.96 & 3.91 & 0.675 & 0.938 & 0.874 \\
\hline & HAP15 & CAATG & 4.26 & 3.82 & 3.53 & 3.61 & 0.354 & 0.839 & \\
\hline
\end{tabular}

Abbreviations: CTR, control; LD, linkage disequilibrium; SCZ, schizophrenia; SNP, single-nucleotide polymorphism.

Block 1: rs13212283-rs538681-rs3757016-rs352068; Block 2: rs13204445-rs12208304-rs6568819-rs10499080. Block 3: rs9488289-rs13209064-rs2243356-rs2499618-rs2025191.

Significant $P$-values are in italic bold. Rare haplotypes are not shown, if frequency $<3 \%$.

Significant $P$-values are in italic
aBased on 10000 permutations.

aBased on 10000 permutations.
based on comparison of frequency distribution of all haplotypes for the combination of SNPs. 


\section{CONFLICT OF INTEREST}

The authors declare no conflict of interest.

\section{ACKNOWLEDGEMENTS}

This research was totally supported by and National Natural Science Foundation of China (no. 81401563), China Postdoctoral Science Foundation Funded Project (nos. T70927 and M532029), PhD Programs Foundation of Ministry of Education of China (no. 2013021120078) and Fundamental Research Funds for the Central Universities (nos. 08142024 and 08143003). The funding sources had no role in the design of this study, the collection, analysis and interpretation of data, the writing of the report or the decision to submit the paper for publication.

1 Perälä, J., Suvisaari, J., Saarni, S. I., Kuoppasalmi, K., Isometsä, E., Pirkola, S. et al Lifetime prevalence of psychotic and bipolar I disorders in a general population. Arch Gen Psychiatry. 64, 19-28 (2007).

2 Do, K. Q. Schizophrenia: genes, environment and neurodevelopment. Rev. Med. Suisse 9, 1674-1677 (2013).

3 Hosak, L. New findings in the genetics of schizophrenia. World J. Psychiatry 3, 57-61 (2013).

4 McGrath, J. J., Mortensen, P. B., Visscher, P. M. \& Wray, N. R. Where GWAS and epidemiology meet: opportunities for the simultaneous study of genetic and environmental risk factors in schizophrenia. Schizophr. Bull. 39, 955-959 (2013).

5 Corvin, A. Schizophrenia at a genetics crossroads: where to now? Schizophr. Bull. 39 490-495 (2013).

6 O'Donovan, M. C., Craddock, N., Norton, N., Williams, H., Peirce, T., Moskvina, V. et al. Identification of loci associated with schizophrenia by genome-wide association and follow-up. Nat. Genet. 40, 1053-1055 (2008).

7 Guan, F., Zhang, C., Wei, S., Zhang, H., Gong, X., Feng, J. et al. Association of PDE4B polymorphisms and schizophrenia in Northwestern Han Chinese. Hum. Genet. 131, 1047-1056 (2012).

8 Guan, F., Zhang, B., Yan, T., Li, L., Liu, F., Li, T. et al. MIR137 gene and target gene CACNA1C of miR-137 contribute to schizophrenia susceptibility in Han Chinese. Schizophr. Res. 152, 97-104 (2014).

9 Guan, F., Wei, S., Zhang, C., Zhang, H., Zhang, B., Xing, B. et al. A population-based association study of $2 \mathrm{q} 32.3$ and $8 \mathrm{q} 21.3$ loci with schizophrenia in Han Chinese. J. Psychiatr. Res. 47, 712-717 (2013).

10 Guan, F., Wei, S., Feng, J., Zhang, C., Xing, B., Zhang, H. et al. Association study of a new schizophrenia susceptibility locus of $10 q 24.32-33$ in a Han Chinese population. Schizophr. Res. 138, 63-68 (2012).

11 Singh, S., Kumar, A., Agarwal, S., Phadke, S. R. \& Jaiswal, Y. Genetic insight of schizophrenia: past and future perspectives. Gene 535, 97-100 (2014).

12 Ma, L., Tang, J., Wang, D., Zhang, W., Liu, W., Wang, D. et al. Evaluating risk loci for schizophrenia distilled from genome-wide association studies in Han Chinese from Central China. Mol. Psychiatry. 18, 638-639 (2013).
13 Petronis, A. The origin of schizophrenia: genetic thesis, epigenetic antithesis, and resolving synthesis. Biol. Psychiatry. 55, 965-970 (2004).

14 Mill, J., Tang, T., Kaminsky, Z., Khare, T., Yazdanpanah, S., Bouchard, L. et al. Epigenomic profiling reveals DNA-methylation changes associated with major psychosis. Am. J. Hum. Genet. 82, 696-711 (2008).

15 Kinoshita, M., Numata, S., Tajima, A., Shimodera, S., Ono, S., Imamura, A. et al. DNA methylation signatures of peripheral leukocytes in schizophrenia. Neuromol. Med. 15 95-101 (2013)

16 Cheng, J., Wang, Y., Zhou, K., Wang, L., Li, J., Zhuang, Q. et al. Male-specific association between dopamine receptor D4 gene methylation and schizophrenia. PloS ONE 9, e89128 (2014).

17 Minucci, S. \& Pelicci, P. G. Histone deacetylase inhibitors and the promise of epigenetic (and more) treatments for cancer. Nat. Rev. Cancer. 6, 38-51 (2006)

18 Kim, D., Frank, C. L., Dobbin, M. M., Tsunemoto, R. K., Tu, W., Peng, P. L. et al. Deregulation of HDAC1 by p25/Cdk5 in neurotoxicity. Neuron 60, 803-817 (2008).

19 Tremolizzo, L., Carboni, G., Ruzicka, W. B., Mitchell, C. P., Sugaya, I., Tueting, P. et al. An epigenetic mouse model for molecular and behavioral neuropathologies related to schizophrenia vulnerability. Proc. Natl Acad. Sci. USA 99, 17095-17100 (2002)

20 Parker, K., Maxson, J., Mooney, A. \& Wiley, E. A. Class I histone deacetylase Thd1p promotes global chromatin condensation in Tetrahymena thermophila. Eukaryot. Cell. 6, 1913-1924 (2007).

21 Han, H., Yu, Y., Shi, J., Yao, Y., Li, W., Kong, N. et al. Associations of histone deacetylase-2 and histone deacetylase-3 genes with schizophrenia in a Chinese population. Asia Pac. Psychiatry 5, 11-16 (2013).

22 Schaid, D. J., Rowland, C. M., Tines, D. E., Jacobson, R. M. \& Poland, G. A. Score tests for association between traits and haplotypes when linkage phase is ambiguous. Am. J. Hum. Genet. 70, 425-434 (2002).

23 Gabriel, S. B., Schaffner, S. F., Nguyen, H., Moore, J. M., Roy, J. \& Blumenstiel, B. et al. The structure of haplotype blocks in the human genome. Science 296, 2225-2229 (2002).

24 Barrett, J. C., Fry, B., Maller, J. \& Daly, M. J. Haploview: analysis and visualization of LD and haplotype maps. Bioinformatics 21, 263-265 (2005).

25 Barrett, R. M. \& Wood, M. A. Beyond transcription factors: the role of chromatin modifying enzymes in regulating transcription required for memory. Learn. Mem. 15, 460-467 (2008)

26 Morris, M. J., Karra, A. S. \& Monteggia, L. M. Histone deacetylases govern cellular mechanisms underlying behavioral and synaptic plasticity in the developing and adult brain. Behav. Pharmacol. 21, 409-419 (2010).

27 Monsey, M. S., Ota, K. T., Akingbade, I. F., Hong, E. S. \& Schafe, G. E. Epigenetic alterations are critical for fear memory consolidation and synaptic plasticity in the lateral amygdala. PIOS ONE 6, e19958 (2011).

28 Montgomery, R. L., Hsieh, J., Barbosa, A. C., Richardson, J. A. \& Olson, E. N. Histone deacetylases 1 and 2 control the progression of neural precursors to neurons during brain development. Proc. Natl Acad. Sci. USA 106, 7876-7881 (2009).

29 Kim, M. S., Akhtar, M. W., Adachi, M., Mahgoub, M., Bassel-Duby, R., Kavalali, E. T. et al. An essential role for histone deacetylase 4 in synaptic plasticity and memory formation. J. Neurosci. 32, 10879-10886 (2012).

30 Chen, Y., Sharma, R. P., Costa, R. H., Costa, E. \& Grayson, D. R. On the epigenetic regulation of the human reelin promoter. Nucleic Acids Res. 30, 2930-2939 (2002).

31 Hyman, S. E. Target practice: HDAC inhibitors for schizophrenia. Nat. Neurosci. 15 $1180-1181$ (2012).

Supplementary Information accompanies the paper on Journal of Human Genetics website (http://www.nature.com/jhg) 